\title{
El niño con dolor de piernas
}

\section{Leg Pain in Children.}

Reyes-Cadena A

\section{INTRODUCCIÓN}

El dolor musculoesquelético representa una de las causas más frecuentes de dolor recurrente en pediatría. En general no existe un antecedente traumático evidente sino que los pacientes presentan síntomas, de manera intermitente, desde tiempo antes de solicitar la evaluación; en estos casos, los niños son llevados a consulta por otro motivo y, una vez allí, sus padres mencionan la presencia de dolor musculoesquelético. Menos frecuentemente el motivo de consulta es cojera o incapacidad funcional de aparición brusca sin antecedente traumático previo.

\section{EPIDEMIOLOGÍA}

La epidemiología del dolor musculoesquelético en pediatría ha sido poco estudiada. Encuestas realizadas en Finlandia o el Reino Unido, en escolares, revelan que alrededor de 15\% de los niños presenta dolor musculoesquelético. En Estados Unidos el dolor músculoesquelético es responsable de $7 \%$ de las consultas de atención primaria pediátrica.

\section{ETIOLOGÍA}

Es fundamental tener presente la edad de los pacientes, ya que patologías que pueden ser frecuentes a una edad resultan excepcionales a otra. Existe una gran variedad de etiologías que lo pueden producir, desde cuadros cuyo pronóstico depende de un diagnóstico precoz, hasta cuadros cuya etiología es benigna y que, a pesar de su larga evolución, tienden a la resolución espontánea (Cuadro 1).
Médico Pediatra Adscrito al servicio de Consulta Externa de Pediatría, Instituto Nacional de Pediatría.

Recibido: 18 de enero del 2016

Aceptado: 18 de abril del 2016

Correspondencia

Dr. Reyes-Cadena Armando

Médico Pediatra Adscrito al servicio de

Consulta Externa de Pediatría.

Instituto Nacional de Pediatría.

Insurgentes Sur 3700-C

Col. Insurgentes Cuicuilco

cadenadr@yahoo.com.mx

Este artículo debe citarse como

Reyes-Cadena A. El niño con dolor de piernas. Acta Pediatr Mex 2016;37(3):183-187. 
Cuadro 1. Etiología del dolor músculoesquelético en pediatría a nivel de atención primaria

\begin{tabular}{ll}
\hline Edad & Causas frecuentes $(\mathbf{1 0} \mathbf{1 0})$ \\
$\begin{array}{l}\text { Preescolares } \\
(3-5 \text { años })\end{array}$ & $\begin{array}{l}\text { Traumatismos } \\
\text { Dolores de crecimiento } \\
\text { Sinovitis transitoria } \\
\text { Hipermovilidad }\end{array}$ \\
$\begin{array}{l}\text { Escolares } \\
\text { (6-9 años) }\end{array}$ & $\begin{array}{l}\text { Etología mecánica } \\
\text { Osteocondrosis (enfermedad de Sever) }\end{array}$ \\
Adolescentes & $\begin{array}{l}\text { Traumatismos } \\
\text { (10-14 años) }\end{array}$ \\
& $\begin{array}{l}\text { Etiología mecánica/síndrome por sobrecarga } \\
\text { Osteocondrosis (Osgood -Schlatter) }\end{array}$
\end{tabular}

\section{EVALUACIÓN}

Debido a que cualquier componente del sistema musculoesquelético puede originar dolor, el primer objetivo a la hora de evaluar un paciente con dolor musculoesquelético es determinar de dónde proviene la sintomatología. Para poder diferenciar unos cuadros de otros es útil obtener de manera sistemática la información clínica que permita determinar: 1) qué pacientes deben derivarse inmediatamente a otros especialistas hospitalarios; 2 ) a quiénes se debe solicitar exploraciones complementarias y 3) quiénes precisan un seguimiento clínico sin otro tipo de intervención.

En la anamnesis se preguntará, además de los antecedentes familiares y personales, por el tiempo de evolución del dolor, ya que esto puede ayudar al diagnóstico. Síntomas de inicio agudo pueden sugerir una enfermedad aguda como artritis séptica, osteomielitis, fractura o malignidad. Se interrogará además por antecedentes traumáticos significativos (se entienden como tales aquellos que producen dolor, impotencia funcional, tumefacción o hematoma inmediatamente después o en las primeras 24 horas después del traumatismo). Es importante averiguar si el dolor es constante o intermitente, los síntomas matutinos que mejoran a través del día son más sugestivos de etiología reumatológica. El dolor después de la actividad es sugestivo de síndrome por sobreuso o fractura por estrés. El dolor que provoca despertar nocturno puede tener una etiología benigna como dolores de crecimiento o etiología más seria como malignidad u osteoma osteoide. En los niños que ya hablan se debe interrogar la localización y tipo de dolor. También se debe constatar la duración de los intervalos sin síntomas y si hubo evaluaciones previas por el mismo motivo u otro similar.

Las patologías de mayor gravedad generalmente causan síntomas sistémicos, por lo cual se debe interrogar acerca de fiebre, así como su tiempo de duración, asociación con rash, pérdida de peso, limitación en la actividad física, hiporexia, cambio en el patrón del sueño (Cuadro 2). A continuación se describen las causas más frecuentes de dolor musculoesquelético en la edad pediátrica (Cuadro 3):

Cuadro 2. Propuesta de obtención sistemática de información clínica en el paciente con dolor musculoesquelético

\begin{tabular}{|c|c|}
\hline \multicolumn{2}{|l|}{ Anamnesis } \\
\hline Fiebre & $\begin{array}{l}\text { Infecciones osteoarticulares: artritis séptica, osteomielitis. } \\
\text { Neoplasias: leucemia, neuroblastoma. } \\
\text { Procesos inflamatorios: artritis idiopática juvenil, lupus eritematoso } \\
\text { sistémico, vasculitis }\end{array}$ \\
\hline \multicolumn{2}{|c|}{$\begin{array}{l}\text { Antecedentes traumáticos } \\
\text { (mecanismo, localización, tiempo transcurrido hasta } \\
\text { la aparición de los síntomas) }\end{array}$} \\
\hline Localización & $\begin{array}{l}\text { Diafisaria: traumatismos musculares, fracturas, tumores óseos. } \\
\text { Metafisaria: epifisiolisis,osteomielitis, tumores. } \\
\text { Articular: artritis (séptica, inflamatoria), trastornos mecánicos. }\end{array}$ \\
\hline Patrón doloroso & $\begin{array}{l}\text { Inflamatorio: rigidez tras reposo prolongado, matutina. } \\
\text { Mecánico: dolor asociado con la actividad física, vespertino. }\end{array}$ \\
\hline
\end{tabular}


Reyes-Cadena A. El niño con dolor de piernas






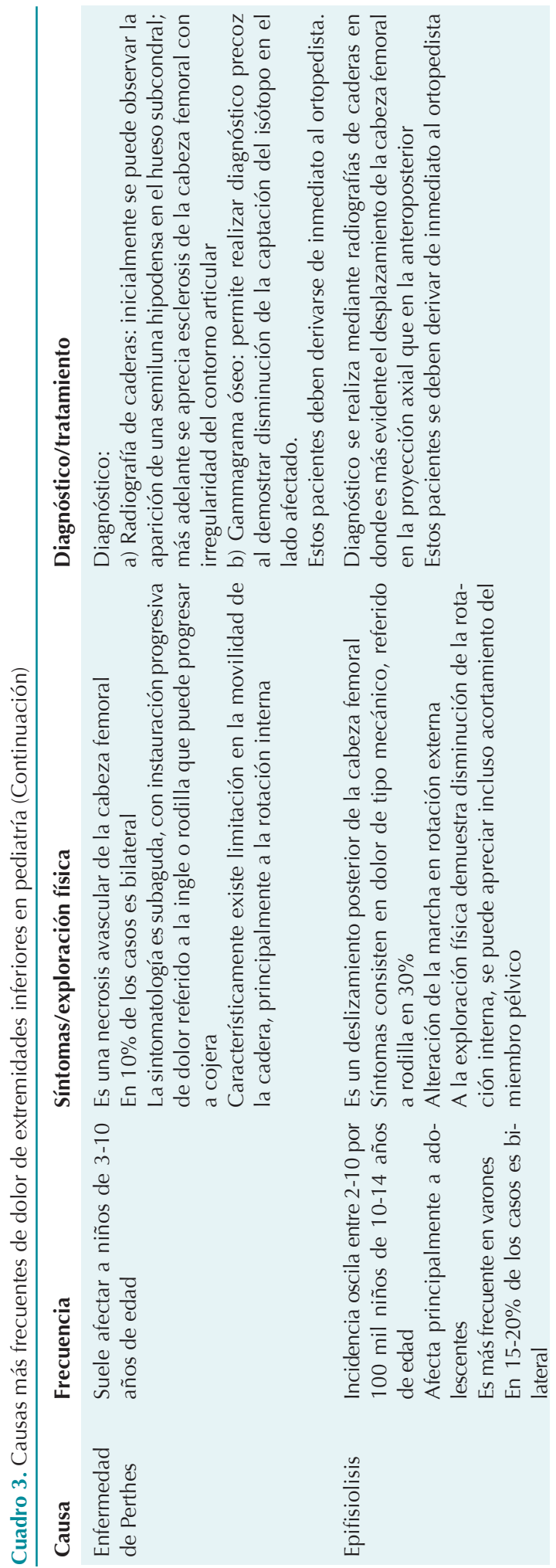

\section{Síndromes por sobrecarga}

Representa un conjunto de procesos producidos por microtraumatismos repetidos. Se observan principalmente en deportistas y durante la edad pediátrica en adolescentes. Puede afectar a músculos (sobrecargas musculares), tendones (epitrocleitis, epicondilitis), fascias (fascitis plantar), huesos (fracturas de estrés) o cartílagos (condromalacia rotuliana).

\section{Neoplasias}

Múltiples tumores pueden producir dolor musculoesquelético. Los más frecuentes son leucemia que puede provocar dolor difuso y artralgias. Habitualmente cursan con sintomatología sistemática y su principal diagnóstico diferencial es la artritis idiopática juvenil sistémica. Otros tumores óseos tanto benignos (osteoma osteoide) como malignos (osteosarcoma, sarcoma de Ewing) pueden provocar dolor esquelético (Cuadro 4).

El dolor músculoesquelético puede estar producido por distintas patologías. Generalmente la etiología es benigna, aunque su diagnóstico diferencial incluye enfermedades cuyo pronós-

Cuadro 4. Hallazgos útiles para distinguir dolor musculoesquelético benigno del potencialmente grave

\section{Dolor benigno}

Alivio con el descanso y empeora con la actividad

Aparición a última hora del día

Dolor nocturno que se alivia Dolor nocturno que no se con analgésicos y masajes

Ausencia de inflamación articular

Hiperlaxitud articular

Ausencia de hipersensibilidad ósea

Fuerza normal

\section{Signos de alarma}

Mejora con la actividad y se presenta durante el reposo

Rigidez matutina Dolor nocturno que no se
alivia con analgésicos y empeora con masaje Presencia de inflamación articular Rigidez articular Hipersensibilidad ósea Debilidad muscular 
Reyes-Cadena A. El niño con dolor de piernas

tico depende en gran medida de un diagnóstico precoz. Una anamnesis minuciosa puede dar claves necesarias para realizar el diagnóstico o para decidir derivar al paciente al especialista adecuado. Sin embargo, el diagnóstico etiológico depende en gran medida de una exploración física detallada.

La utilidad de las exploraciones complementarias en atención primaria se limita al hemograma y reactantes de fase aguda en caso de sospecha de neoplasias o infecciones osteoarticulares y a las radiografías, en caso de fracturas, patología ortopédica y tumores óseos.

\section{Lecturas recomendadas}

1. De Inocencio-Arocena J. Dolor musculoesquelético en pediatría de atención primaria. Etiología y orientación diagnóstica. Arch argent pediatr 2006;104(3):275-283.

2. López-Robledillo J.C. Síndrome de dolor musculoesquelético en la edad pediátrica. Pediatr Integral 2013;XVII(1):15-23.

3. M.L.-Tse S,M. Laxer.R. Approach to Acute Limb Pain in Childhood. Pediatrics in Review May 2006;27(5):170-180.

4. Gedalia A. Joint Pain in children: An Algorithmic Approach. IMAJ 2002;4:837-842. 